\title{
Segmentation of Noisy Images Using the Rank M-Type L-Filter and the Fuzzy C-Means Clustering Algorithm
}

\author{
Dante Mújica-Vargas, Francisco J. Gallegos-Funes, and Rene Cruz-Santiago
}

Mechanical and Electrical Engineering Higher School

National Polytechnic Institute of Mexico

Av. IPN s/n, Edificio Z, acceso 3, $3^{\text {er }}$ piso; SEPI-Electronica, Col. Lindavista, 07738, México

D. F. México, Phone/Fax: (5255)57296000 ext. 54622

\begin{abstract}
In this paper we present an image processing scheme to segment noisy images based on a robust estimator in the filtering stage and the standard Fuzzy C-Means (FCM) clustering algorithm to segment the images. The main objective of paper is to evaluate the performance of the Rank M-type L-filter with different influence functions and to establish a reference base to include the filter in the objective function of FCM algorithm in a future work. The filter uses the Rank M-type (RM) estimator in the scheme of L-filter, to get more robustness in the presence of different types of noises and a combination of them. Tests were made on synthetic and real images subjected to three types of noise and the results are compared with six reference modified Fuzzy C-Means methods to segment noisy images.
\end{abstract}

Keywords: robust estimators, RM-estimator, L-filter, Fuzzy C-Means, segmentation, noise.

\section{Introduction}

Image segmentation is a key step forward image analysis and serves in a variety of applications, including pattern recognition, object detection, medical imaging, robot vision, military surveillance [1]. Image segmentation can be defined as the partition of an image into different meaningful regions with homogenous features using discontinuities or similarities of the image such as intensity, color, texture, and so on [2]. Numerous segmentation techniques have been developed and reported in the literature.

Fuzzy clustering as a soft segmentation method has been widely studied and successfully applied to image segmentation. Among the fuzzy clustering methods, Fuzzy C-Means (FCM) [3] algorithm is the most popular method because it is simple, easy to program, and can retain much more information than hard methods. Although fuzzy clustering methods work well on most noise-free images, they have a serious limitation: they do not incorporate any information about spatial context, which cause them sensitivity to the noise or outliers data. Then it is necessary to modify the objective function to incorporate local information of the image to get better results. 
Following the image processing chain [4] to get a good segmentation stage, is necessary to have a good pre-filtering stage. The filter must be robust at the presence of different levels and types of noise, and in the extreme case, when the image is not noisy, the filtering stage does not distort the image in any way. Taking into consideration the above, in this paper we use a RML-estimator [5] to perform image filtering under the conditions mentioned the presence or absence of noise.

The outline of this paper is as follows. Section 2 presents information about the M, $\mathrm{R}$ and $\mathrm{L}$ estimators, and how to merge them. Section 3 gives a recall of the standard Fuzzy C-Means clustering algorithm. Experimental results are compared with some reference methods are shown in section 4. Finally, some conclusions are drawn in section 5 .

\section{Proposed Method}

To segment noisy images we use the image processing chain shown in Figure 1. In this one the segmentation is the central point and crucial for next stages, and therefore it is necessary to have good results at this stage, but the segmentation has a total dependency of the early filter stage. The propose method is given by the RMLestimator in the filtering stage and then to segment by the standard Fuzzy C-Means clustering algorithm.

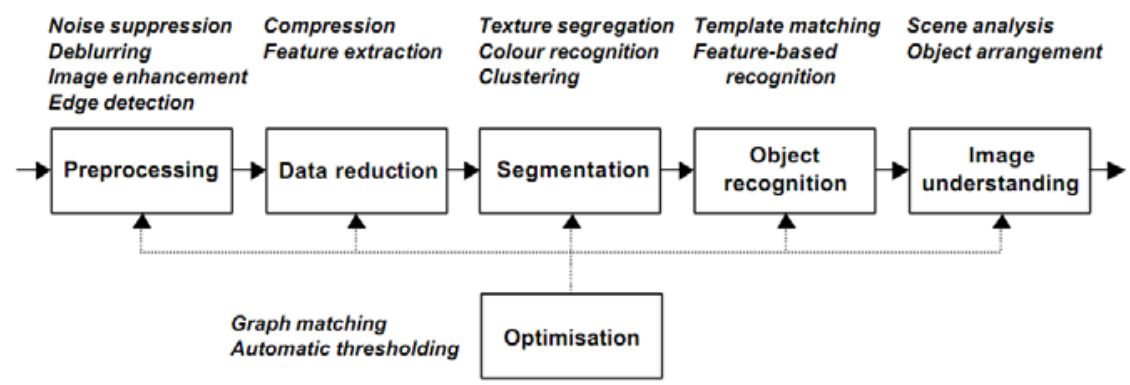

Fig. 1. The image processing chain containing the five different tasks: preprocessing, data reduction, segmentation, object recognition and image understanding

\subsection{RM-Estimator}

The R-estimators form a class of nonparametric robust estimators based on rank calculations $[6,7,8,9]$. They are known to be robust estimators and are used in the signal processing area like R-filters. The median estimator (median filter) is the best choice when any a priori information about data $X_{i}$ distribution shape and its moments is unavailable [7],

$$
\hat{\theta}_{\text {med }}=\left\{x_{(n+1 / 2)}, \quad \text { for odd } n\right\}
$$

where, $x_{(i)}$ is the element with $i$ rank, $n$ is the size of the sample, and $1 \leq i \leq n$. 
M-estimators are a generalization of maximum likelihood estimation (MLE) and were proposed by Peter Hubert $[6,7,8,9]$. Their definition is given by a robust loss function $\rho(x)=\ln (f(x))$, connected with the probability density function $f(x)$ for the sample data $x_{i}, i=i, \ldots, n$. The objective of M-estimators is to find an estimation $\widehat{\theta}$ of $\theta$ such that,

$$
\hat{\theta}=\underset{\theta \in \Theta}{\arg \min }\left(\sum_{i=1}^{n} \rho\left(x_{i}-\theta\right)\right)
$$

The estimation of the localization parameter $\theta$ can be found by calculating the partial derivative of $\rho$ (with respect to $\theta$ ) introducing the function,

$$
\begin{gathered}
\psi(x, \theta)=\frac{\partial}{\partial \theta} \rho(x, \theta) \\
\sum_{i=1}^{n} \psi\left(x_{i}-\theta\right)=0
\end{gathered}
$$

The robust M-estimator solution for $\theta$ is determined by imposing certain restrictions on the influence function $\psi(x)$ (see Table 1) or the $\operatorname{samples}\left(x_{i}-\theta\right.$ ), called censorization or trimming. The standard technique used to calculate the $\mathrm{M}$ estimation is based on the iterative Newton method, but can be simplified by a singlestep algorithm [9] to calculate the lowered M-estimate of the average $\theta$ value,

$$
\theta_{M}=\frac{\sum_{i=1}^{n} x_{i} \tilde{\psi}\left(x_{i}-\operatorname{med}\left\{x_{n}\right\}\right)}{\sum_{i=1}^{n} 1_{[-r, r]} \widetilde{\psi^{\prime}}\left(x_{i}-\operatorname{med}\left\{x_{n}\right\}\right)}
$$

where, $\tilde{\psi}$ is the normalized influence function $\psi: \psi(x)=x \tilde{\psi}(x)$. Is evident that (4) represents the arithmetic average of $\sum_{i=1}^{n} \psi\left(x_{i}-\operatorname{med}\left\{x_{n}\right\}\right)=0$, which is evaluated on the interval $[-r, r], r$ is the parameter connected with restrictions on the range of $\psi$. The simplest restriction on the range of $\psi$ is the limit of the Huber's estimator $\tilde{\psi}_{r}(X)=\min (r, \max (X, r))=[X]_{-r}^{r}[9]$.

Table 1. Influence functions used in the proposed filter

\begin{tabular}{crrr}
\hline Influence function & \multicolumn{1}{c}{ Formulae } \\
\hline simple cut & $\psi_{\text {cut }(r)}(x)$ & $= \begin{cases}x, & |x| \leq r \\
0, & \text { otherwise }\end{cases}$ \\
Andrew's sine & $\psi_{\sin (r)}(x)$ & $=\left\{\begin{array}{cc}\sin (x / r), & |x| \leq r \pi \\
0, & \text { otherwise }\end{array}\right.$ \\
Tukey's biweight & $\psi_{b i(r)}(x)$ & $= \begin{cases}{\left[1-\left(\frac{x}{r}\right)^{2}\right]^{2},} & |x| \leq r \pi \\
0, & \text { otherwise }\end{cases}$ \\
Hampel's three part redescending & $\psi_{\alpha, \beta(r)}(x)$ & $=\left\{\begin{array}{cc}x, & 0 \leq|x|<\alpha \\
\alpha \operatorname{sgn}(x), & \alpha \leq|x|<\beta \\
\alpha \frac{r-|x|}{r-\beta} \operatorname{sgn}(x), & \beta \leq|x|<r \\
0, & r \leq|x|\end{array}\right.$
\end{tabular}


The proposal to enhancement the robust properties of M-estimator (4) by using the R-estimator (1) consists of the application procedure similar to the median average instead of arithmetic one [9],

$$
\theta_{\text {medM }}=\operatorname{med}\left\{x_{i} \psi\left(x_{i}-\operatorname{med}\left\{x_{n}\right\}\right)\right\}
$$

The properties of the RM-estimator (5) are increased by fusing the ability of the Restimator to suppress the impulsive noise and the use of the different influence functions of the M-estimator to provide more robustness. Thus, it is expected that the combined RM-estimator can be better than the originals R-estimator and M-estimator.

\subsection{RML- Estimator}

We propose to use the RM-estimators into the linear combinations of order statistics defined by the L-filter. The proposed RM L (Rank M-type L) -filter employs the idea of RM-KNN algorithm [9]. The following representation of L-filter is often used [6],

$$
\theta_{L}=\sum_{i=1}^{n} a_{i} x_{(i)} \text { with } a_{i}=\int_{\frac{i-1}{n}}^{\frac{i}{n}} h(\lambda) d \lambda / \int_{0}^{1} h(\lambda) d \lambda
$$

where, $x_{(i)}, i=1, \ldots, n$ are the data sample and $a_{i}, i=1, \ldots, n$ are the weighted coefficients of filter, $h(\lambda)$ is the noise probability distribution function defined in $[0,1] \rightarrow R$, which satisfice $\int_{0}^{1} h(\lambda) d \lambda \neq 0$, then, the L-filters satisfice the equation $\sum_{i=1}^{n} a_{i}=1$ [7]. Using this method the weighted coefficients can be computed for different distribution functions (Exponential, Laplacian, Uniform, etc.) and windows sizes, we used a $3 \times 3$ window because we obtained the best results and more detail preservation than $5 \times 5$ or $7 \times 7$ windows.

To introduce the RM-estimator in the scheme of L-filter, we should to present the ordered data sample of L-filter as function of an influence function. For this reason, the L-filter (6) is writing as [10,11]:

$$
\begin{gathered}
\theta_{L}=\sum_{i=1}^{n} a_{i} \psi\left(x_{i}\right) x_{i} \\
\psi\left(x_{i}\right)=\left\{\begin{array}{cc}
1 & i \leq(2 l+1)^{2} \\
0 & \text { otherwise }
\end{array}\right.
\end{gathered}
$$

where, $\psi(x)$ is the influence function used in the L-filter, $\psi\left(x_{i}\right) x_{i}$ is the ordered data sample according with the eq. (6), and $(2 l+1)^{2}$ is the filtering window size. Then, the new filter can be obtained by merging the L-filter (7) and the RM-estimator (5). The Median M-type L (MML) -filter can be expressed by [10,11]:

$$
\theta_{M M L}=\frac{\operatorname{med}\left\{a_{i}\left[x_{i} \psi\left(x_{i}-\operatorname{med}\left\{x_{n}\right\}\right)\right]\right\}}{a_{\text {med }}}
$$

where, $\theta_{M M L}$ is the output MML- filter, $x_{i} \psi\left(x_{i}-\operatorname{med}\left\{x_{n}\right\}\right)$ are the selected pixels in accordance with the influence function in the sliding filter window, and $a_{m e d}$ is the median of coefficients $a_{i}$ used as scale constant. 
To improve the properties of impulsive noise suppression of the proposed filter we introduce an impulse detector, this one chooses that pixel is or not filter. The impulsive detector used is defined as [12]:

$$
\left[\left(\operatorname{rank}\left(x_{i j}\right) \leq s\right) \vee\left(\operatorname{rank}\left(x_{i j}\right) \geq N-s\right)\right] \wedge\left(\left|x_{i j}-\operatorname{med}\left\{x_{n}\right\}\right| \geq U_{2}\right)
$$

where, $x_{i j}$ is the central pixel in the filtering window, $s>0$ y $U_{2} \geq 0$ are thresholds, $N$ is the length of the data and $\operatorname{med}\left\{x_{n}\right\}$ is the median of pixels into the filtering window.

\section{Classic Fuzzy C-Means Clustering}

Fuzzy C-Means is a method for data classification, where each data belongs to a cluster to some degree, which is specified by a membership value [3]. This algorithm performs the iteration of two indispensable conditions to minimize the following objective function,

$$
J_{f}\left(X ; U_{f}, C\right)=\sum_{i=1}^{N} \sum_{j=1}^{c} u_{i j}^{m} d_{i j}^{2} \text { subject to } \sum_{i=1}^{N} u_{i j}>0 \quad \forall_{j} \text { and } \sum_{j=1}^{c} u_{i j}=1 \quad \forall_{i}
$$

where, $X=\left\{x_{i} \mid i=1, \ldots, N\right\}$ denotes the set of $N$ feature vectors, $c$ is the number of classes, $m \in[1, \infty)$ is a weighting exponent called the fuzzifier, $d_{i j}^{2}$ is the distance from feature vector $x_{i}$ to the center of the class $c_{j}$ and $C=\left(c_{1}, \ldots, c_{j}\right)$ is a vector with all center classes. $U=\left[u_{i j}\right]$ is a $N x c$ matrix denoting the constrained fuzzy cpartition. The value of $u_{i j}$ denotes the degree of membership of $x_{i}$ to the class $c_{j}$. Taking in account both constrains, the membership matrix and the cluster prototypes can be calculated with the following equations [13],

$$
\begin{gathered}
u_{i j}=\frac{1}{\sum_{k=1}^{c}\left(\frac{d_{i j}^{2}}{d_{i k}^{2}}\right)^{\frac{1}{m-1}}} \\
c_{j}=\frac{\sum_{i=1}^{N} u_{i j}^{m} x_{i}}{\sum_{i=1}^{N} u_{i j}^{m}}
\end{gathered}
$$

\section{Experimental Results}

The performance of the proposed method was tested on synthetic and real images. In both cases the quantitative results were compared with FCM_S1, FCM_S2, EnFCM, FGFCMS1, FGFCM_S2 and the FGFCM algorithms taken from [14]. The comparison is done by the optimal segmentation accuracy (SA), where SA is defined as the number of correctly classified pixels divided by sum of the total number of pixels [14]. 


\subsection{Results on a Synthetic Image}

The algorithms were applied on the synthetic image as shown in Figure 2(a) (128x128 pixels, two classes with two gray levels taken as 0 and 90) corrupted by different levels of Gaussian and Salt \& Pepper noise and a mixed noise of Gaussian white noise $N(0,100)$ and unit dispersion, cero centered symmetric $\alpha$-stable $(S \alpha S)$ noise; for all algorithms $c=2$, according to [14] $\lambda_{g}=6$ and $\alpha S=3.8, r=5$ for all RML_FCM algorithms, $\alpha=0.16 r, \beta=0.8 r$ for Hampel influence function, $s=4$ and $U_{2}=5$ for the impulse detector. Table 2 and 3 show the SAs for the comparative and proposed algorithms, respectively, on the synthetic images and Figure 2 depicts the visual results.

Table 2. SA \% of six reference algorithms on synthetic image

\begin{tabular}{cccccccccc}
\hline \multirow{2}{*}{ Algorithm } & Gaussian & Gaussian & Gaussian & S\&P & S\&P & S\&P & mixed & mixed & mixed \\
& $3 \%$ & $5 \%$ & $8 \%$ & $5 \%$ & $10 \%$ & $15 \%$ & $\alpha=0.3$ & $\alpha=0.5$ & $\alpha=0.7$ \\
\hline FCM_S1 & 99.14 & 96.42 & 92.32 & 98.69 & 97.14 & 94.78 & 93.80 & 98.68 & 99.59 \\
FCM_S2 & 98.78 & 96.12 & 92.23 & 98.77 & 97.54 & 95.98 & 97.25 & 99.27 & 99.79 \\
EnFCM & 99.50 & 97.65 & 94.62 & 98.05 & 94.77 & 94.94 & 95.34 & 99.09 & 99.69 \\
FGFCM_S1 & 99.57 & 98.20 & 95.41 & 99.07 & 96.47 & 92.40 & 95.82 & 99.44 & 99.83 \\
FGFCM_S2 & 99.13 & 96.82 & 93.12 & 99.99 & 99.98 & 99.84 & 99.65 & 99.97 & 100.00 \\
FGCM & 99.51 & 98.10 & 95.10 & 99.91 & 99.47 & 98.36 & 97.95 & 99.84 & 99.96 \\
\hline
\end{tabular}

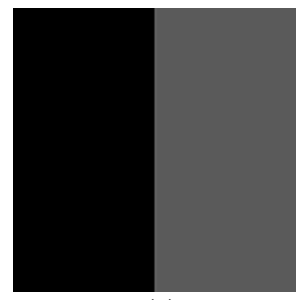

(a)

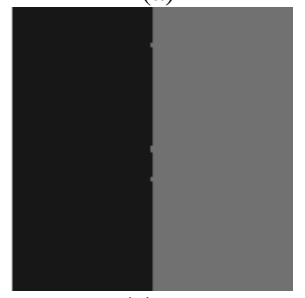

(e)

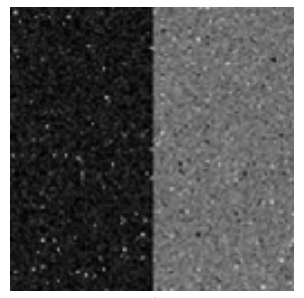

(b)

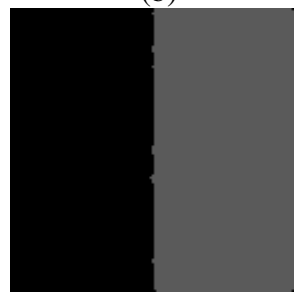

(f)

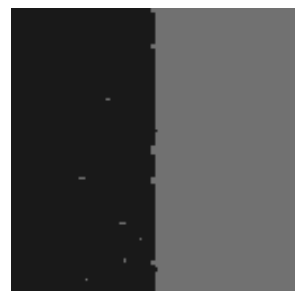

(c)

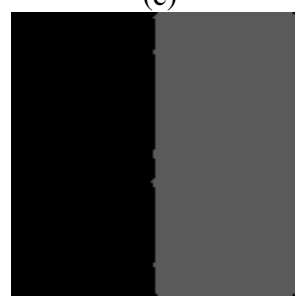

(g)

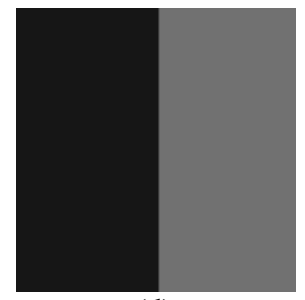

(d)

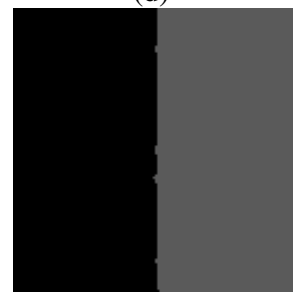

(h)

Fig. 2. Segmentation results in a synthetic image. (a) Original image. (b) Noisy image. (c) FGFCM_S1. (d) FGFCM_S2. (e) FGFCM. (f) RML_FCM H,U. (g) RML_FCM H,E. (h) RML_FCM H,L., where Hampel's three part redescending (H), Uniform (U), Exponential (E), Laplacian (L). 
Table 3. SA \% of RML_FCM algorithms on synthetic image

\begin{tabular}{|c|c|c|c|c|c|c|c|c|c|c|}
\hline $\begin{array}{l}\text { Influence } \\
\text { function }\end{array}$ & $\begin{array}{l}\text { RML } \\
\text { distribution } \\
\text { filter }\end{array}$ & $\begin{array}{c}\text { Gaussian } \\
3 \%\end{array}$ & $\begin{array}{c}\text { Gaussian } \\
5 \%\end{array}$ & $\begin{array}{c}\text { Gaussian } \\
8 \%\end{array}$ & $\begin{array}{l}\text { S\&P } \\
5 \%\end{array}$ & $\begin{array}{l}\text { S\&P } \\
10 \%\end{array}$ & $\begin{array}{l}\text { S\&P } \\
15 \%\end{array}$ & $\begin{array}{l}\text { mixed } \\
\alpha=0.3\end{array}$ & $\begin{array}{l}\text { mixed } \\
\alpha=0.5\end{array}$ & $\begin{array}{l}\text { mixed } \\
\alpha=0.7\end{array}$ \\
\hline \multirow{3}{*}{$\begin{array}{l}\text { Simple } \\
\text { cut }\end{array}$} & & & 99.94 & 99.92 & 99.97 & 99.95 & 99.74 & 99.93 & 99.86 & 99.82 \\
\hline & Exp & & & & 97 & & 9.74 & & & 9.82 \\
\hline & & & & & 9.97 & 5 & 99.74 & & 9.89 & 99.84 \\
\hline \multirow{3}{*}{$\begin{array}{l}\text { Andrew's } \\
\text { sine }\end{array}$} & & & 95 & 99.9 & 99.95 & 99.94 & 99.92 & 99.90 & 99.85 & 99.80 \\
\hline & Expo & & & 99.9 & 99.95 & 99.94 & 99.92 & 99.92 & 99.88 & 99.83 \\
\hline & Lapl & & & 95 & 99.95 & 99.94 & 99.92 & 99.89 & 99.86 & 99.81 \\
\hline \multirow{3}{*}{$\begin{array}{l}\text { Tukey's } \\
\text { biweight }\end{array}$} & nifo & 9 & & 99.9 & 99.93 & 99.88 & 99.86 & 99.89 & 99.84 & 99.78 \\
\hline & Expon & 99.9 & & 99.92 & 99.93 & 99.88 & 99.86 & 99.88 & 99.84 & 99.74 \\
\hline & Laplacian & & & 99.92 & 99.93 & 99.88 & 99.86 & 99.91 & 99.86 & 99.79 \\
\hline \multirow{3}{*}{$\begin{array}{l}\text { Hampel's } \\
\text { three part } \\
\text { redesding }\end{array}$} & niform & 99.95 & 99.93 & 99.93 & 99.97 & 99.79 & 99.70 & 99.92 & 99.85 & 99.79 \\
\hline & Exponential & & & & 99.97 & 99.79 & 99.70 & 99.92 & 99.88 & 99.81 \\
\hline & Laplacian & 99.95 & 99.93 & 99.93 & 99.97 & 99.79 & 99.70 & 99.91 & 99.86 & 99.80 \\
\hline
\end{tabular}

\subsection{Results on a Real Image}

The robustness on a real image was tested using the eight real image corrupted by a mixed noise. The original image (Figure 3(a), 308x242 pixels) was corrupted simultaneously by Gaussian white noise $N(0,180)$ and unit dispersion, zero centered symmetric $\alpha(\alpha=0.9)$-stable $(S \alpha S)$ noise. For all algorithms $c=3$, according to [14] $\lambda_{g}=2$ and $\alpha=8$ for reference algorithms, $r=5$ for all RML_FCM algorithms, $\alpha=0.16 r$, $\beta=0.8 r$ for Hampel influence function, $s=4$ and $U_{2}=5$ for the impulse detector. Table 4 gives the SAs for all algorithms on the eight image and Figure 3 presents the visual results.

Table 4. SA \% of reference and RML_FCM algorithms on a real image

\begin{tabular}{cccc}
\hline Influence function & RML distribution filter & Algorithm & SA \% \\
\hline- & - & FCM_S1 & 88.91 \\
- & - & FCM_S2 & 88.64 \\
- & - & EnFCM & 82.18 \\
- & - & FGFCM_S1 & 82.18 \\
- & - & FGFCM_S2 & 89.11 \\
- & - & FGFCM & 91.87 \\
Simple cut & Uniform & RML_FCM & 90.52 \\
& Exponential & RML_FCM & 86.28 \\
& Laplacian & RML_FCM & 90.21 \\
Andrew's sine & Uniform & RML_FCM & 89.92 \\
& Exponential & RML_FCM & 88.86 \\
& Laplacian & RML_FCM & 89.67 \\
Tukey biweight & Uniform & RML_FCM & 90.47 \\
& Exponential & RML_FCM & 90.09 \\
& Laplacian & RML_FCM & 89.96 \\
Hampel & Uniform & RML_FCM & 89.51 \\
& Exponential & RML_FCM & 89.58 \\
& Laplacian & RML_FCM & 89.10 \\
\hline
\end{tabular}




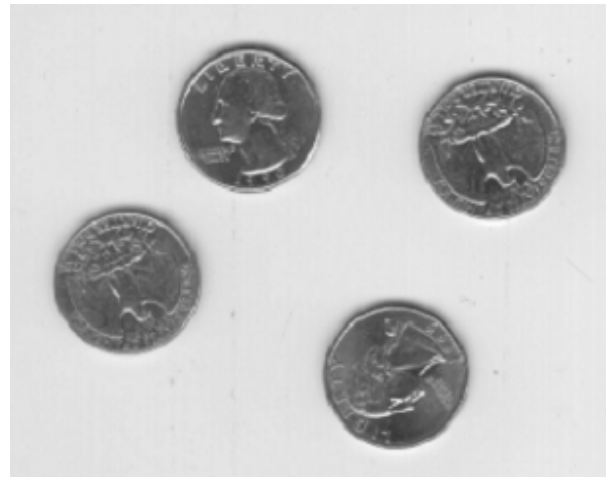

(a)

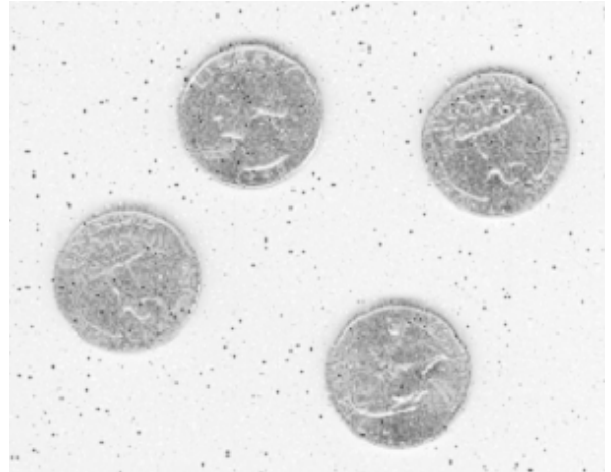

(c)

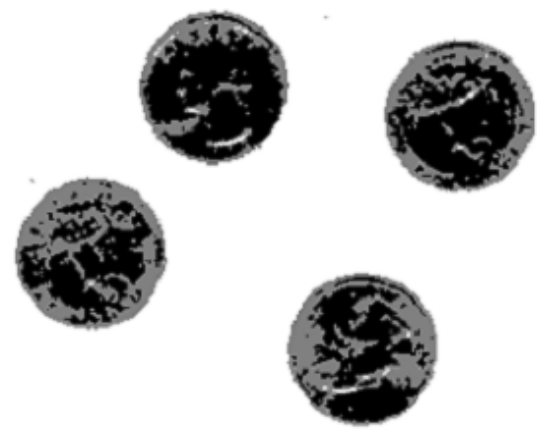

(e)

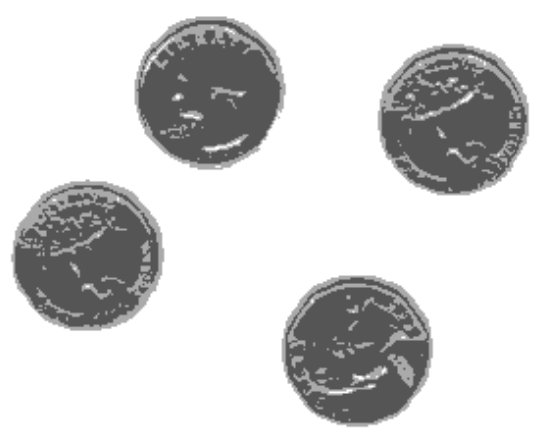

(b)

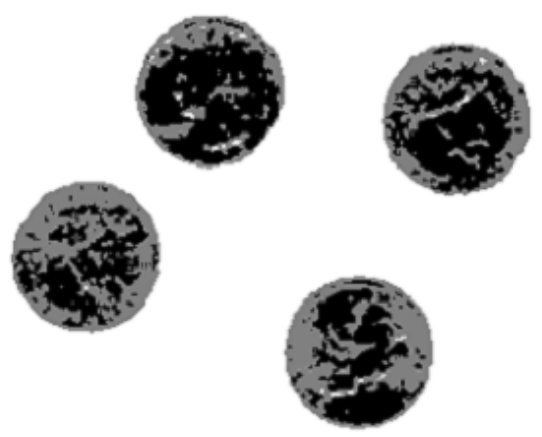

(d)

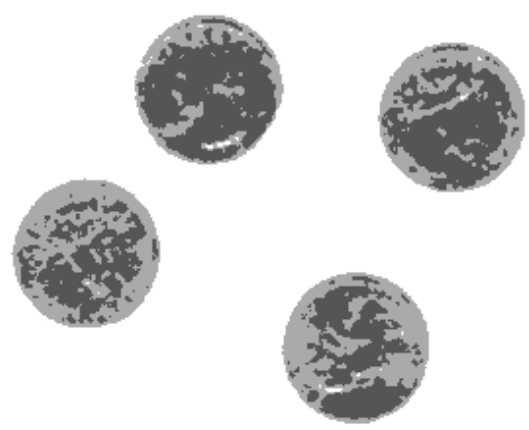

(f)

Fig. 3. Segmentation results on a real image. (a) Original image. (b) Original image segmentation. (c) Noisy image. (d) FGFCM_S2. (e) FGFCM. (f) RML_FCM H,U. (g) RML_FCM H,E. (h) RML_FCM H,L., where Hampel's three part redescending (H), Uniform (U), Exponential (E), Laplacian (L). 


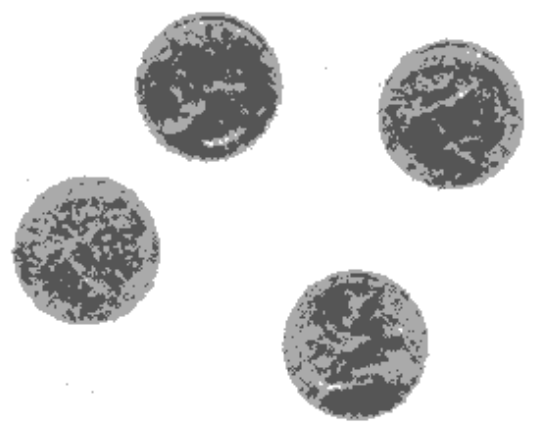

(g)

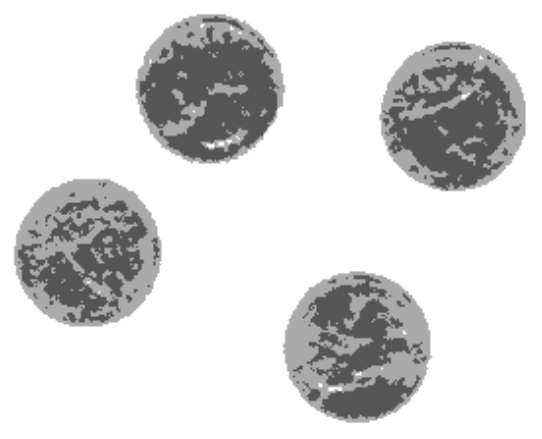

(h)

Fig. 3. (continued)

\section{Discussion of Results}

In tests in a synthetic image, one can see that the performance of the procedure RML_FCM presents better performance in the case of images corrupted by only one type of noise, but when the image has mixed noise the ability of method is comparable with the different reference algorithms. We must stress that although these algorithms include local information is the FGFCM algorithm the most robust in the presence of noise or outliers in the image. One can see that on a real image tests, the RML_FCM presents a SA\% higher than the other algorithms. In the case of algorithms RML_FCM in all variations their performance is even close to FGFCM algorithm.

\section{Conclusions}

This paper presented the robust RM L-filters designed with different influence functions. These filters were the base of the FCM to segment noisy images. The performance of proposed RML_FCM is better than the comparative methods. To improve the properties of the FCM algorithm to segment free or noisy images as future work, the RM L-filter will be included in the function cost of the FCM algorithm to modify it. Besides, the segmentation will be extended on color images.

\section{Acknowledgments}

This work is supported by National Polytechnic Institute of Mexico and Conacyt. 


\section{References}

1. Kim, J., Fisher, J.W., Yezzi, A., Cetin, M., Willsky, A.S.: A nonparametric statistical method for image segmentation using information theory and curve evolution. IEEE Transactions on Image Processing, 1486-1502 (2005)

2. Dong, G., Xie, M.: Color clustering a nd learning for image segmentation based on neural networks. IEEE Transactions on Neural Networks 16(4), 925-936 (2005)

3. Bezdek, J.C.: Pattern Recognition with Fuzzy Objective Function Algorithms. Plenum Press, New York (1981)

4. Egmont-Petersen, M., de Ridder, D., Handels, H.: Image processing with neural networks - a review. Institute of Information and Computing, Utrecht University, Utrecht (2002)

5. Gallegos-Funes, F.J., Linares, R., Ponomaryov, V., Cruz-Santiago, R.: Real-Time image processing using the Rank M-type L-filter. Cientific 11, 189-198 (2007)

6. Pitas, A.N.V.: Nonlinear Digital Filters. Kluwer Academic Publishers, Boston (1990)

7. Astola, J., Kousmanen, P.: Fundamentals of Nonlinear Digital FIltering. CRC Press, Boca Raton (1997)

8. Hampel, F.R., Ronchetti, E.M., Rouseew, P.J., Stahel, W.A.: Robust Statistics. In: The approach based on influence function. Wiley, NY (1986)

9. Gallegos-Funes, F.J., Ponomaryov, V.: Real-time image filtering scheme based on robust estimators in presence de noise impulsive. Real Time Imaging 8(2), 78-90 (2004)

10. Gallegos-Funes, F.J., Varela-Benitez, J.L., Ponomaryov, V.: Real-time image processing based on robust linear combinations of order statistics. In: Proc. SPIE Real-Time Image Processing, vol. 6063, pp. 177-187 (2006)

11. Varela-Benitez, J.L., Gallegos-Funes, F.J., Ponomaryov, V.: RML-filters for real rime imaging. In: Proc. IEEE $15^{\text {th }}$ International Conference on Computing, CIC 2006, pp. 43-48 (2006)

12. Aizenberg, L., Astola, J., Breging, T., Butakoff, C., Egiazarian, K., Paily, D.: Detectors of the impulsive noise and new effective filters for the impulse noise reduction. In: Proc. SPIE Image Processing, Algorithms and Systems II, vol. 5014, pp. 410-428 (2003)

13. Theodoridis, S., Koutroumbas, K.: Pattern Recognition, 4th edn. Elsevier, Amsterdam (2009)

14. Cai, W.L., Chen, S.C., Zhang, D.Q.: Fast and robust fuzzy c-means clustering algorithms incorporating local information for image segmentation. Pattern Recognition 40, 383-825 (2007) 